\title{
Vermischte spanische Beiträge.
}

\author{
I. Span. estantigua.
}

Man hat dieses Wort teils von estatua antigua (so Paul Foerster, Span. Sprachl. S. 232), teils von *stantifica (G. Baist in dieser Ztschr. V 243) herleiten wollen. Es scheint aber als hueste antigua zu erklären zu sein, wie folgende Stelle aus dem Tractado del calor natural von Francisco de Villalobos (Obras, Çaragoça I 544, f. XXIXv) zeigt:

"No sabemos si es alguna fantasma que aparece a vnos y no a otros como trasgo: o como la hueste antigua."1

In der asturischen Volksüberlieferung bezeichnet übrigens noch heut zu Tage hueste. (güeste, huestia, huesstiga) soviel als eine nächtliche Prozession von Verstorbenen, siehe z. B. Fermin Canella, Estudios asturianos (Oviedo I 886) p. 133 und Gumersindo Laverde, Apuntes lexicográficos sobre una rama del dialecto asturiano (in der Revista de Asturias III) s. v. güeste.

\section{Einige Bemerkungen zu Gessners Abhandlung über das Altleonesische.}

In seiner trefflichen Studie über El libro de Alexandre (Romania 1875) macht Morel-Fatio auf die von Gessner mit Schweigen übergangenen höchst interessanten Diphthongformen uo ua aufmerksam, die im Alexandre (nur ein Beispiel: muobre) und in den Concilbestimmungen bei Muñoz (uorto buonas, muarto buanas nuastra) vorkommen. $\mathrm{Zu}$ den von M.-F. angeführten Beispielen aus dem Leonerconcil ist hinzuzufügen, dafs die Form uorto dreimal vorkommt (auf der Seite 77 zweimal und einmal S. 85) und ferner die von ihm nicht bemerkte Form fuara S. 88.

1 Die ältesten Belegstellen, die ich für das Wort gefunden sind übrigens folgende aus der ersten Hälfte des I6. Jahrh.:

Válala el diablo á estd vieja, con qué viene como estantigua á tal hora (La Celestina, Acto VII).

Andar de estantigua y fantasma de noche (Comedia Seraphina, Colecc. de libros esp. raros ó curiosos T. V S. 380).

¿Quién es esta fantasma 6 estantigua P (Comedia llamada Selvagia, ibid. S. I36). 
Bei Durchblätterung des Fuero Juzgo (Ausg. der span. Akademie von 1815) habe ich nun noch folgende Beispiele dieser Diphthongformen angetroffen:

$\begin{array}{lrrl}\text { S. } 1, \text { var. } 23 & \text { nuova } \\ " & 3, & 22 & \text { luogo } \\ " & 4, & 26 & \text { oabras } \\ " 18, & 2 & \text { ruogo } \\ " & 23, & 20 & \text { puode } \\ " & 23, & 23 & \text { voaltas }\end{array}$

S. 35, var. 5 puoda
$\Rightarrow 76,, 21$ avnola
$" 96, " 21$ encuantra
$" 105, " 15$ aguaradores
$" 105, " 22$ aguoradores
$" 135, "$ I I tuorto

Über das Vorkommen eines uó- uá- klingenden Diphthongs im heutigen Asturischen vergleiche übrigens meine Abhandlung Anteckningar om folkmålet $i$ en trakt af vestra Asturien (Upsala r 887$)$ S. I 5.

In einer Variante zum Fuero Juzgo (S. 109,12) findet man die von Gefsner nicht bemerkte interessante Form dido (vgl. ital. dito, Grundris I 507) für dedo. Dieselbe Form siddo, nebst dida (Zehe), habe ich im Südwestasturischen angetroffen (ygl. Ant. S. 25).

Die in den altleonesischen und altasturischen Denkmalen so häufig vorkommenden Formen dia die estia estie für den Konjunktiv Präs. werden von Gefsner S. 28 angeführt. S. 32 Anm. 42 vermutet er dafs man diá estiá zu lesen habe: durch uncastilianische Diphthongierung der regelmälsigen Formen dé esté, meint er, habe man zunächst dié estié gebildet, dann durch Vokalentartung diá estiá - eine höchst unwahrscheinliche Entwickelung.

Gefsner bemerkt auch dafs dia auch im Altportugiesischen vorkommt und citiert aus dem Elucidario eine Stelle aus einer alten Verordnung der in äufsersten Norden liegenden Stadt Bragança die Form wäre demnach nur nordportugiesisch.

Aufserdem hat man nun auch im Gallicischen dia estia nebst dea estea, in Bercianischen ${ }^{1}$ dia estia, im modernen Asturischen central, dea estea und südwestlich día dié und estía estié (vgl. Ant. S. 49), welche letzteren Formen also den alten leon. und ast. am nächsten kommen.

Wahrscheinlich ist nun dafs die Formen dia dea, estia estea einen uralten Typus (*deam etc.) repräsentieren und dafs man immer día estía, niemals aber wie Gefsner meint, diá estiá gesprochen. Aus día estía entwickelten sich zunächst durch Vokalschwächung díe estíe (wie hacie aus hacía), aus denen dié estié durch Accentvorrückung hervorgehen könnten (vgl. in einer alten Romanze ponien hacien mit bien Belen reimend, siehe Diez, Gramm. 5 S. 326), was mutmafslich am leichtesten in einer Fürwort oder

1 Ensayos poéticos en dial. berciano von Fernandez y Morales. Der s. g. bercianische Dialekt ist übrigens bekanntlich nur ein mit castilianischen Elementen versetztes Gallicisch. 
Endung tragenden Penultima vor sich ging (díame $>$ díeme $>$ diéme). $\mathrm{Ob}$ man übrigens in den Denkmalen díe oder dié zu lesen habe, ist nicht leicht zu entscheiden: wahrscheinlich kamen wohl beide Betonungen vor; im Südwestast. hörte ich stets dié- in der Penultima, sonst día.

Seite 32 s. v. enton führt Gefsner aus dem Fuero Juzgo I 15 die Form pia (cast. pié) an. Dieselbe Form kommt auch F. J. 77 und 109 (pias, zweimal) und auf der genannten Seite I 5 dreimal vor, also 6 Beispiele (aus zwei Handschriften). Analog dieser Form ist das von Gefsner nicht bemerkte ia (= est) F. J. I I 5 aus eben der Handschrift, die 4 von den 6 pia giebt; hierzu auch ya (= est) F. J. 16 aus derselben Handschrift.

Sowohl pia als (y)ia, nebst (y)ie, kommen nun auch im Westasturischen vor (vgl. Ant. S. 38), wo auch $y a$ (=et) gesagt wird (in alten leon. und ast. Texten ie hie = et).

Die Formen pía und ía (so waren gewifs auch die altleon. Formen betont) müssen wohl zunächst aus píe und ie hervorgegangen sein (vgl. día $<$ diem), die ihrerseits durch Accentverschiebung aus pié und ié entsprungen sein können (wobei bemerkt werden kann dals -ié auslautend überhaupt ungewöhnlich war und ist); falls der Diphthong ié aus einem älteren ie entwickelt ist könnten pía und ia wohl auch ein letzter Rest dieser ursprünglichen Betonung sein, die sich hier auslautend erhalten hätte. Das südwestasturische ya (= et) ist wohl in Betracht seiner Unbetontheit, direkt aus ye ié hervorgegangen.

Eine von Gefsner mit Schweigen übergangene aber sehr charakteristische Erscheinung in den altleonesischen und asturischen Denkmalen ist die Prothese von $d$ in mit al- beginnenden Fürwörtern. Im Fuero Juzgo findet man aus vier verschiedenen Codices dalguno dalgun dalguna dalgunos dalgunas (S. V, XIII, 7, 11, I2, 30, 45, I41, 155), España Sagrada T. XXXVI dalguno dalguna (S. 142, 143) und altasturisch bei Fernandez-Guedra dalquien dalguien (S. 71,72 ). Im modernen Asturischen scheinen ähnliche Formen allgemein zu sein: so findet man in der das asturische Centrum vorwiegend repräsentierende Bablelitteratur dalgun dangun daquien (vgl. altast. dalquien) daqut. ('etwas', das ein aliquid zu sein scheint; vgl. prov. alque), ferner dacuando ('jemals', das auf aliquando weist) und dayure dayures (aliubi, vgl. unten); im Südwestasturischen hörte ich dalgun daquien daque (d. i. dáque, nicht daqué wie in der Bablelitteratur) dacuando; auch in der Provinz Santander ('La Montaña') scheinen diese Formen zu Hause zu sein: in den Escenas montañesas von Pereda habe ich zwei daque gefunden (und hier adjektivisch: daque cargo 'irgend ein Auftrag').

In den entsprechenden verneinenden Wörtern kommt nun ferner ein das $\mathrm{n}$ - ersetzendes $\mathrm{d}$ - vor: so degun deguna im Fuero Juzgo 
(S. I 46, I 49, beide aus derselben Handschrift). Altasturisch habe ich nur nengun gefunden, aber im modernen Asturischen findet man in der Bablelitteratur dengun delgun und denyure denyures (port. nenhures) und in Südwestasturien hörte ich oft dengun; endlich legt Pereda denguno in den Mund seiner Montañeses. Ferner kommt denguno auch andalusisch vor, und catalanisch dengú degú dingú, provençalisch degun.

Das prothetische $d$ der angeführten bejahenden Wörter kann nun natürlich nicht denselben Ursprung wie das $d-=n-$ der verneinenden Formen haben; wohl aber ist analogische Anbildung von algun etc. an ein auf irgend einer Weise entstandenes dengun, oder umgekehrt, möglich. Dengun scheint eine weit gröfsere örtliche Verbreitung als dalgun etc. zu haben und könnte demnach wohl auch Ansprüche auf Priorität erheben; es scheint auch leichter als dalgun eine nicht allzu unwahrscheinliche Erklärung zu gestatten.

Diez, E. W. IIc s. v. degun (prov. und altleon.), nimmt an, es sei diese Form dem ahd. dihein nachgebildet; Schuchardt (Die Cantes Flamencos, in dieser Zeitschr. V) meint, dafs denguno durch Dissimilation aus nenguno entstanden (wobei Combinationen wie. nen nengun, sin nengun, en nengun der Dissimilation besonders förderlich sein mufsten, vgl. auch span. péndola = pennola). Die letztere Erklärung scheint sehr annehmbar, und einem auf dieser Weise entstandenen dengun könnte nun das leonesisch-asturische dalgun etc. wohl nachgebildet sein. Hierbei ist aber zu erinnern, dafs ich in den alten Denkmalen nur die zwei oben citierten altleon. degun aus einer Handschrift des Fuero Juzgo gefunden, während ich überall anders, und altasturisch ausschliefslich, nur nengun gesehen habe. Allerdings weifs ich, falls dalgun dem dengun nicht nachgebildet wäre, keine bessere Erklärung dafür vorzuschlagen.

In seinem Wörterbuch IIb führt Diez das Wort alubre aus dem Fuero Juzgo (offenbar aus dem Glossar) an nebst dem gleichwertigen a iubre (d. i. ayubre) aus Berceo Loores $\mathrm{II}_{4}=$ aliubi. Gelsner führt das Wort nicht an.

Im Fuero Juzgo S. 147 findet man nun im Texte $a$ iubre und in den Varianten alubre alumbre allum allure aliur und endlich aimbre (Cod. Toled.), das ohne Zweifel eine Fehlschreibung ist (dem castilianischen Schreiber dieser Handschrift war das Wort vermutlich völlig unbekannt). Das Wort, das bekanntlich auch altund neuportugiesich ist - alhures alhur (nebst algures, algur = alicubi nach Diez E. W.) und dazu verneinend nenhures - findet sich auch alt- und neuasturisch: alluri in den Ordenanzas de Oviedo aus dem I3. Jahrh. (Sangrador y Vitores, Historia de la Administracion etc. del Principado de Asturias S. 38I) und in der Bablelitteratur dayure dayures endayures (mit prothetischem $\mathrm{d}$, vgl. oben) verneinend denyure denyures; im südwestlichen Asturien notierte ich zufälliger Weise nur das verneinende nichuris. 
In der Gramm. 5 S. 744 setzt nun Diez, wie im E. W., das altspanische alubre = aliubi, meint aber dals das port. alhures, alhur auf aliorsum weise ( $=$ fr. ailleurs etc.), das doch regelrecht nur ein port. *alhuso geben konnte (wie altport. suso juso $=$ susum deusum); allerdings citiert Reinhardstöttner (Port. Gramm. S. 252) auch ein alhus aus dem Elucidario, das dem aliorsum besser entspricht. Wahrscheinlich sind doch wohl altspan. alubre aiubre etc. vom port. alhures alhur nicht $\mathrm{zu}$ trennen, und das Etymon aliubi (aljube aljubre aljure $+s$ ) scheint dem aliorsum vorzuziehen $\mathrm{zu}$ sein.

$\mathrm{Da}$ algures nur portugiesisch vorzukommen scheint, könnte in Frage gestellt werden, ob man hierin wirklich, wie Diez will, ein alicubi zu sehen habe, oder ob nicht vielmehr dies algures, als eine Mischform von alhures und algum $\mathrm{zu}$ betrachten wäre.

Ebenso, da das ch des südwestasturischen nichuris nichts anders als ein vom bejahenden *achuris gekommenes ursprüngliches lj sein kann, ist es wahrscheinlich, dafs auch das port. nenhures unter Mitwirkung von nenhum frei auf alhures gebildet ist, und nicht etwa, wie Diez (E. W. und Gramm. S. 744) vermutet, aus nec ubi entsprungen.

Åke W:SON Munthe. 